\title{
The cytological and molecular role of DOMAINS REARRANGED METHYLTRANSFERASE3 in RNA- dependent DNA methylation of Arabidopsis thaliana
}

Pedro Costa-Nunes ${ }^{1}$, Ji Young Kim² ${ }^{2}$ Evelyn Hong ${ }^{2}$ and Olga Pontes ${ }^{1,3^{*}}$

\begin{abstract}
Background: Plants have evolved a unique epigenetic process to target DNA cytosine methylation: RNA-directed DNA methylation (RdDM). During RdDM, small RNAs (smRNAs) guide methylation of homologous DNA loci. In Arabidopsis thaliana, the de novo DNA methyltransferase that ultimately methylates cytosines guided by smRNAs in all sequence contexts is DOMAINS REARRANGED METHYLTRANSFERASE 2 (DRM2). Recent reports have shown that DRM2 requires the catalytic mutated paralog DRM3 to exert its function through a still largely unknown process. To shed light on how DRM3 affects RdDM, we have further characterized its role at the molecular and cytological levels.
\end{abstract}

Findings: Although DRM3 is not required for RdDM loci transcriptional silencing, it specifically affects loci's DNA methylation. Interestingly, DRM3 and DRM2 regulate the DNA methylation in a subset of loci differently. Fluorescence In Situ Hybridization and immunolocalization analyses showed that DRM3 is not required for the large-scale nuclear organization of heterochromatin during interphase, with the notable exception of the $45 \mathrm{~S}$ ribosomal RNA loci. DRM3 localizes exclusively to the nucleus and is enriched in a round-shaped domain located in the nucleolar periphery, in which it colocalizes with components of the RdDM pathway.

Conclusions: Our analyses reinforce the previously proposed chaperone role of DRM3 in RdDM. Overall, our work further demonstrates that DRM3 most likely functions exclusively with DRM2 in RdDM and not with other $A$. thaliana DNA methyltransferases. However, DRM3's regulation of DNA methylation is likely target- or chromatin context-dependent. DRM3 hypothetically acts in RdDM either upstream of DRM2, or in a parallel step.

Keywords: Small RNAs, DNA methylation, DRM3, Nuclear localization, Interphase organization

\section{Findings \\ Background}

Eukaryotes modulate gene expression via histone posttranslational modifications and/or DNA cytosine methylation. Repressive epigenetic marks, such as deacetylated histone tails and dimethylated lysine 9 of histone H3 (H3K9me2), are characteristic of silent chromatin, i.e. heterochromatin. Conversely, acetylated histones and H3

\footnotetext{
* Correspondence: opontes@unm.edu

'Department of Biology, University of New Mexico, MSC03 2020, 1 University of New Mexico, Albuquerque, NM 87131, USA

${ }^{3}$ Present address: Shanghai Center for Plant Stress Biology, Chinese Academy of Sciences, 3888 Chenhua Road, Shanghai 201602, P. R. China

Full list of author information is available at the end of the article
}

lysine 4 trimethylation (H3K4me3) are hallmarks of transcriptionally permissive chromatin, the euchromatic portion of the genome. In regards to DNA cytosine methylation, hypermethylation is usually associated with heterochromatin, while hypomethylation is associated with euchromatin.

In Arabidopsis thaliana, DNA methylation stably suppresses the activity of potentially deleterious transposons and other mobile elements throughout cellular division [1]. The $A$. thaliana genome encodes ten predicted cytosine methyltransferase genes, which can be clustered into three distinct groups according to their similarities with mammalian DNA methyltransferases 
[2]. METHYLTRANSFERASE1 (MET1) is the Dnmt1 ortholog responsible for maintenance methylation in CG motifs [3]. Symmetrical DNA methylation in the CHG context (in which $\mathrm{H}=\mathrm{A}, \mathrm{T}$ or $\mathrm{C}$ ) is achieved by activity of CHROMOMETHYLASE3 (CMT3) [4], a member of the plant-specific CMT family. CMT2 is responsible for asymmetric methylation $(\mathrm{CHH})$ at histone $\mathrm{H} 1$-enriched heterochromatic regions, binding to $\mathrm{H} 3 \mathrm{~K} 9 \mathrm{me} 2$ [5]: a process facilitated by the chromatin remodeler DECREASED DNA METHYLATION 1 (DDM1) [6].

Finally, $A$. thaliana orthologs of the mammalian Dnmt3 family include DOMAINS REARRANGED METHYLTRANSFERASE1-3 (DRM1-3). DRM1 expression is restricted to the mature egg cell, where together with DRM2, it functions as a de novo methyltransferase [7]. 24-nucleotide (nt) short interfering RNAs (siRNAs) guide DRM2 activity in RNA-directed DNA methylation (RdDM), to methylate de novo asymmetric cytosines $(\mathrm{CHH})[8,9]$.

DRM3 is another member of the DRM family in $A$. thaliana that has been implicated in RdDM [10]. DRM3 is a catalytically mutated DRM2 paralog, as its Motif IV carries an S585N amino acid substitution. Intriguingly, although bearing a mutation in its catalytic domain, DRM3 is required to establish $\mathrm{CHH}$ DNA methylation at some RdDM target loci, such as AtSNI, MEA-ISR and FWA, and to accumulate $5 \mathrm{~S}$ ribosomal RNA (rRNA) homologous siRNAs $[10,11]$. Despite these effects, DRM3 cannot compensate for the loss of DRM2 and it is proposed that they function together at the step of catalysis to establish DNA methylation [10], in a mechanism not very well understood.

Given the importance of DNA methylation for regulating gene expression, which in turn affects organisms' phenotype, the goal of this study was to deepen our understanding of how this epigenetic mark is established. Specifically, we sought to clarify the role of DRM3 in RdDM by determining whether DRM3 partners exclusively with DRM2 and how DRM3 relates to other fundamental components of the RdDM pathway. To that end, we characterized DRM3's effects on several RdDM targets at both the transcriptional and DNA methylation levels. We also determined DRM3's effect on nuclear organization during interphase, as well as its cellular localization.

\section{Results and discussion}

DRM3 is required for 24-nt siRNA accumulation but does not significantly contribute to transcriptional silencing at RdDM targets

As mentioned previously, DRM3 is proposed to act as a chaperone of DRM2 activity in RdDM [10], which is a complex process requiring several protein complexes. In short, siRNA biogenesis in $A$. thaliana results from the combined activity of the plant-specific RNA polymerase IV (Pol IV) and RNA-DEPENDENT RNA POLYMERASE 2 (RDR2) [12,13]. Downstream of this process, the lead strand of the siRNA duplex is loaded to an Argonaute (AGO) protein, usually AGO4 [14,15]. AGO4 can be crosslinked to noncoding transcripts of the plant-specific RNA polymerase $\mathrm{V}$ (Pol V), suggesting that co-transcriptional Pol V-dependent interactions account for AGO4-siRNA recruitment to target loci [16]. Finally, these AGO4-siRNA complexes, through interactions with other pathway members, direct DRM2 and possibly DRM3 activities $[9,10]$, resulting in the establishment of DNA methylation and repressive histone modifications to silence the corresponding genomic region $[16,17]$.

To reveal whether other RdDM targets are also regulated by DRM3, we began by characterizing the effect of drm3 on 24-nt siRNA production (Figure 1A) by RNA Northern blot. A significant reduction in its abundance relative to wild type (WT) was observed in 5S rRNA (siR1003), in agreement with [10]; similar reductions in AtSNI and Copia repeats were also observed.

In RdDM, loss of DNA methylation and siRNA production correlates with transcriptional reactivation at a given target locus. Semi-quantitative RT-PCR (Reverse Transcription Polymerase Chain reaction) of RdDM targets, such as AtSNI, soloLTR and IG/LINE, revealed that transcriptional reactivation occurs in $d r m 3$ mutants at lower levels than in nrpd1, nrpe1 (Pol IV and V's largest subunits, respectively) or $d r m 2$ genetic mutants (Figure $1 \mathrm{~B}$, C). Negligible effects on the transcriptional reactivation of AtGP1, LTRCO1 and LTRCO3 in drm3 mutants were also observed (Figure 1C). Furthermore, DRM3 is not required for transcriptional regulation of AtMuI, Athila, Ta3 and CACTA transposable elements (not shown) under MET1 control [18,19], nor of the CMT3-regulated At2g34655 loci (Figure 1C). These observations indicate that DRM3 is likely RdDM-specific, as according to our data, DRM3 is not required to silence MET1 and CMT3-dependent loci and because it functions together with DRM2 in RdDM dependent transcriptional silencing, as previously reported [10].

\section{DRM3 differentially affects DNA methylation at RdDM target loci}

We next evaluated cytosine methylation at $\mathrm{CHH}$ sequence contexts by using a Methylation Sensitive PCR (MSP) assay. This assay allows the methylation status of cytosines to be evaluated at particular sequence motifs, by taking advantage of methylation-sensitive restriction enzymes. Methylated cytosines at the restriction enzyme recognition site will block endonuclease activity and allow for the PCR amplification, whereas DNA cleavage will occur at unmethylated sites and no PCR product will be observed. This MSP analysis revealed that for AtSNI, solo LTR and LTR1 and LTR3 
A)

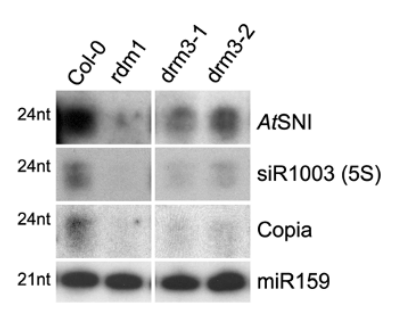

C)

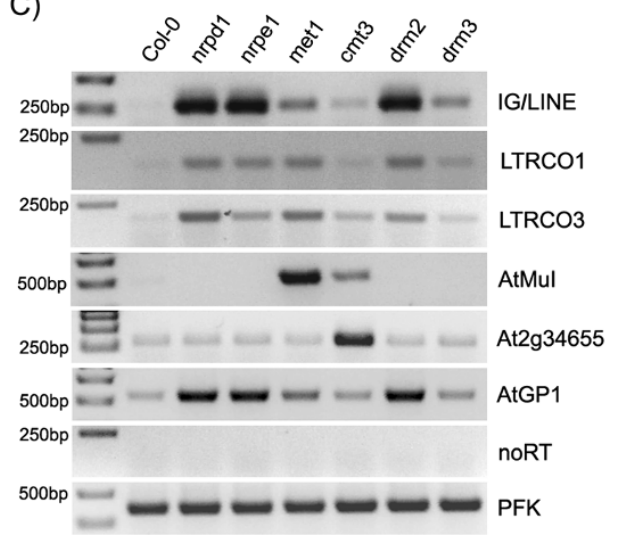

B)

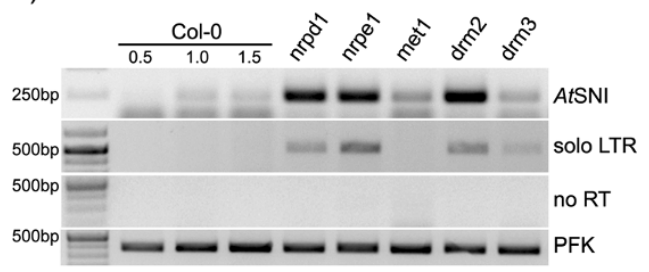

D)

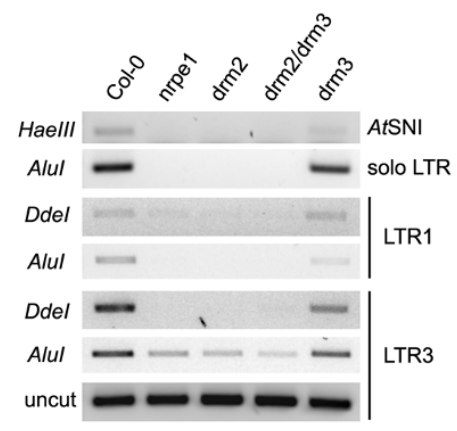

Figure 1 Effect of $d r m 3$ loss of function in siRNA accumulation, transcriptional activity and DNA methylation. (A) DRM3 is required for the accumulation of repeat-derived 24-nt smRNAs. SmRNA Northern blot showing reduced $24 \mathrm{nt}$ smRNA accumulation in a drm3 mutant background relative to Col-0 (WT), as also observed for rdm1 and drm2. (B)(C) RT-PCR analysis shows that the drm3 mutation has only a minor effect on transcriptional reactivation of RdDM targets, when compared to other bona fide pathway members. Loci under the transcriptional control of MET1 or CMT3 do not require DRM3 activity (AtMul, At2g34655). (D) Methylation Sensitive PCR (MSP) assay showing that CHH methylation is not severely compromised in loss of function drm3 mutants relative to drm2 and nrper mutants in RdDM-controlled loci.

regions of LTRCO1 and LTRCO3 transposons, which are single copy members of the LTRCO Copia-like retrotransposon family in A. thaliana [20], methylation was essentially unaffected in the drm3 background (Figure 1D). In contrast, these transposable elements show extensive demethylation in nrpe1, drm2 and the $d r m 2 / d r m 3$ double mutant at discrete cytosines (Figure 1D). Our MSP results correlate DNA demethylation with the transcriptional activity of RdDM targets (Figure $1 \mathrm{~B}, \mathrm{C}$ ), with $d r m 3$ displaying lower transcriptional reactivation levels than nrpe1, nrpd1 and drm2 mutants. DRM2 methylation at these loci was only slightly affected in a drm3 background, implying that DRM2 does not strictly require DRM3 at all chromosomal locations.

To obtain deeper insights into how DRM3 regulates DNA methylation, we performed bisulfite sequencing at RdDM-controlled loci, namely simpleHAT2, MEA-ISR, AtSNI, siRNA02 and IGN5. No significant differences in cytosine methylation levels were found at the simpleHAT2 locus between $d r m 2$ and $d r m 3$ (not shown). Our $M E A-I S R$ bisulfite sequencing results (Figure 2A) are in agreement with Henderson et al. (2010): while drm2 displayed 100\% DNA methylation loss at $\mathrm{CHH}$ sites, the drm3 mutant showed partial demethylation in $20 \%$ of the amplicons relative to WT (Additional file 1: Figure S1).
Thus, the DRM3-dependent DNA methylation phenotype results from the differential regulation of a subset of target sequences at the MEA-ISR loci.

DNA methylation analysis of AtSNI loci by bisulfite sequencing showed a high degree of similarity between $d r m 2$ and $d r m 3$, with an overall loss of $\mathrm{CHH}$ methylation and reduction at non- $\mathrm{CHH}$ sites (Additional file 1: Figure S2). However, a subset of non- $\mathrm{CHH}$ cytosines sites was decreased in the drm 3 and nrpe1 mutant backgrounds, but not in drm2 (Figure 2B). Also, marked differences between drm2 and drm3 were found at siRNA02 loci, where hypermethylation at CG sites was observed in nrpe1 and drm2, but not in drm 3 nor in the $d r m 2 / d r m 3$ double mutant (Figure 2C). At IGN5, drm2 and drm3 mutations did not affect CG methylation and only slightly affected CHG sites compared to WT. In contrast, significantly fewer methylated cytosines were observed in a $\mathrm{CHH}$ context in both mutant lines (Figure 2D) relative to WT. Interestingly, while in the drm2 background, $\mathrm{CHH}$ methylation was almost totally eliminated throughout the full length of the IGN5 sequence; in drm3, the 5 ' end still displayed significant levels of DNA methylation compared to WT (Figure 2D, 2E). Taken together, these bisulfite analyses suggest that DRM3 affects DNA methylation differently than DRM2 


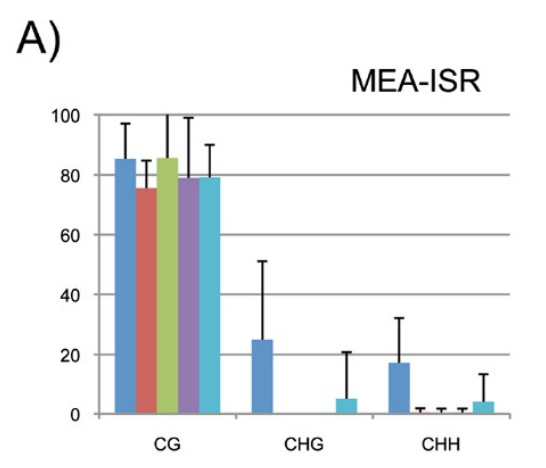

B)

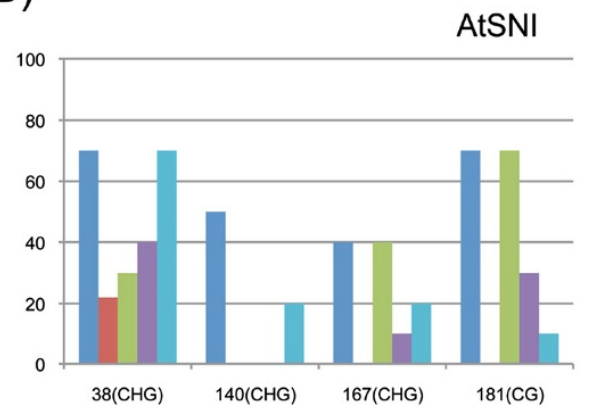

C)

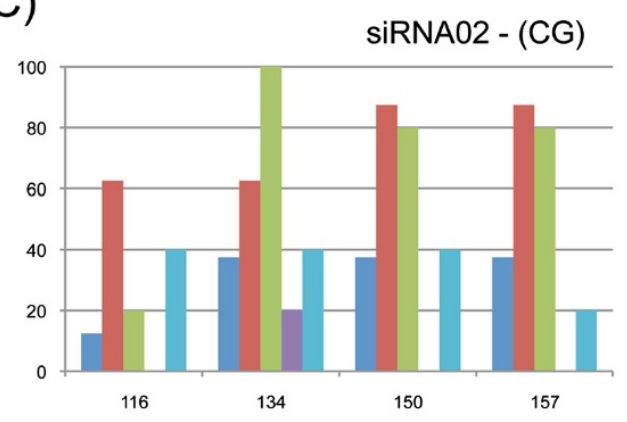

D)

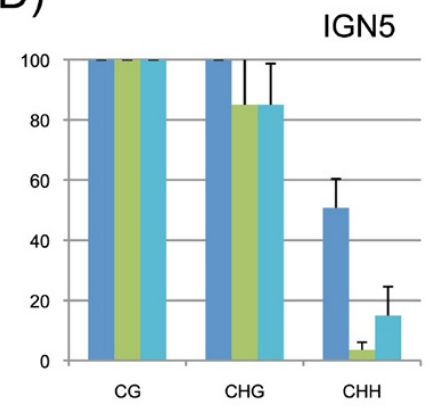

E)

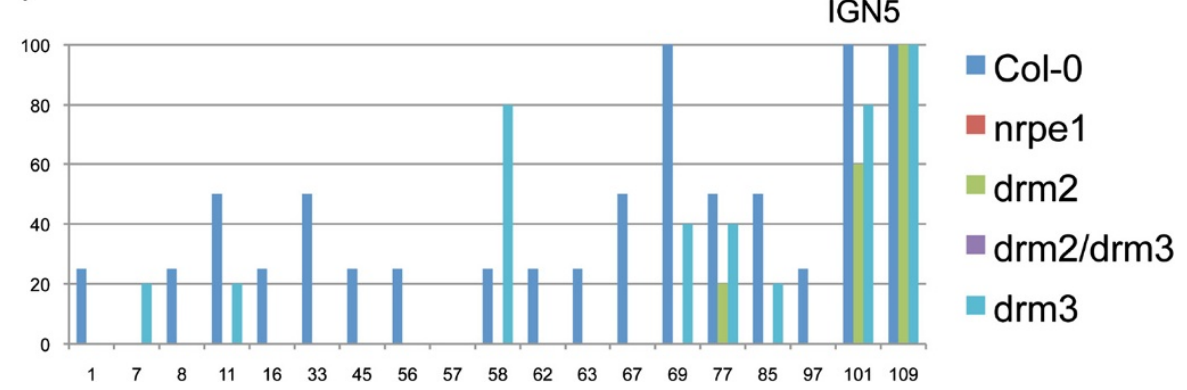

Figure 2 Bisulfite sequencing analysis at distinct RdDM targets in drm3 mutant. (A) Frequency of cytosine methylation according to sequence context at MEA-ISR loci. A significant reduction of methylation at non-CG sites was observed in drm3 relative to WT, but not to the levels observed for drm2. Frequency of symmetrical site methylation at (B) AtSNI and (C) siRNA02 (CG) are differentially affected by drm2 and drm3. (D) Loss of CHH methylation at IGN5 is higher in a drm2 background relative to drm3, especially at its $5^{\prime}$ end (E). All cytosines are in a CHH context except at positions $101(\mathrm{CHG})$ and 109 (CG) (E). Where present, error bars refer to standard deviation. $y$-axis: frequency (\%); $x$-axis: numbers correspond to cytosine position.

at specific loci, and that this difference is likely influenced by chromosome/chromatin location and target loci.

\section{DRM3 associates with AGO4 at the nucleolar periphery}

It has been previously shown that the RdDM pathway is localized in distinct nuclear and cytoplasmic subcompartments [21,22]. For instance, Pol IV is strictly localized to the nucleoplasm, whereas RDR2 also accumulates in a distinct nuclear domain in the perinucleolar periphery [21,23-25]. Proteins like RDM1, required for Pol V activity and targeting of DNA methylation, and AGO4 are also found in this nuclear domain. Importantly, mislocalization of the upstream RdDM components required for siRNA production and proper targeting of de novo DNA methylation affects the nuclear localization of downstream pathway members involved mainly in the targeting process [21]. In addition, active site mutants of Pol V's largest subunit, NRPE1, abrogate DNA methylation at RdDM targets and disrupt the nuclear localization of the polymerase [26]. Together, this indicates that the cellular localization of RdDM pathway components is linked to their function. Therefore, determining the nuclear localization pattern of an $\mathrm{RdDM}$ pathway member and how its loss of function affects the nuclear organization of other proteins can yield important clues to determine at which step of the pathway 
it functions, namely, if it is required for siRNA biogenesis or involved in DNA methylation targeting [21].

To learn more about DRM3's cellular localization and its relationship to other pathway members, we generated transgenic lines with an N-YFP DRM3 cDNA construct driven by a $35 \mathrm{~S}$ promoter. The transgene was able to restore DNA methylation levels when introduced into a drm3-1 background at both AtSNI loci [10] and in the LTR3 region, demonstrating that the transgene is functional (Figure 3A).

DRM3 localizes throughout the nucleoplasm and was found to accumulate preferentially in a perinucleolar focus (Figure $3 \mathrm{~B}$ ), in a pattern similar to the one observed for RdDM pathway members RDM1 and AGO4 $[21,23,25]$. The DRM3 interphase localization pattern was not disrupted when the transgene was introduced into drm2, ago4 and nrpe1 mutant backgrounds (Figure 3C), nor was the nuclear localization of AGO4 and DRM2 disrupted in a drm3 background (Additional file 1: Figure S3). Interestingly, co-immunolocalization in interphase nuclei expressing YFP-DRM3 and AGO4, using an antibody specific to the native protein, showed that both proteins colocalized in a perinucleolar domain (Figure 3D), placing DRM3 in the hypothetical smRNA processing center previously described [21,24].
Altogether, these observations suggest that DRM3 acts downstream of siRNA production in RdDM, as it is nonessential for the localization of the main components of RdDM. Nevertheless, due to its accumulation in the smRNA processing center, it is possible that DRM3 might act upstream of DRM2 in the pathway, in accordance with its proposed chaperone role for DRM2 [10]. The mammalian homologs of DRM2 and DRM3 (Dnmt3a and Dnmt3L, respectively) are known to interact, with the recruitment of Dnmt3a to target loci being dependent on the association of the non-catalytic Dnmt3L with chromatin [27,28]. Given that DRM3 localizes with AGO4 in the same nuclear domain where other RdDM components are also enriched (e.g. RDM1) [25], one could speculate that DRM3 may interact with other proteins or be required for complex assembly.

\section{DRM3 is required for NOR nuclear organization during interphase}

The spatial organization of the genome in the interphase nucleus depends on several factors, including DNA sequence, gene activity and external (a)biotic factors $[29,30]$. Several studies have revealed that cytologically visible heterochromatin formation requires DNA methylation at CG sites and methylation of H3K9 maintained by MET1 and

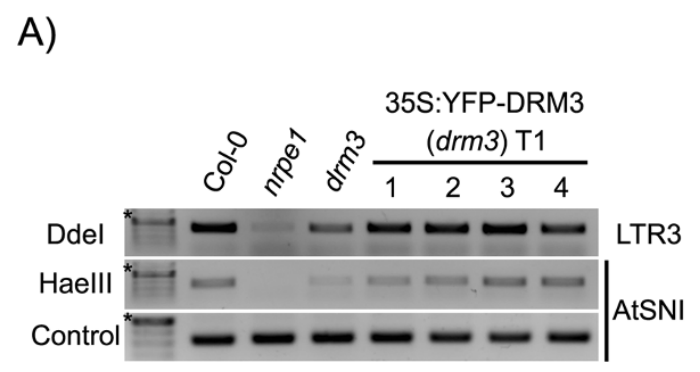

C)

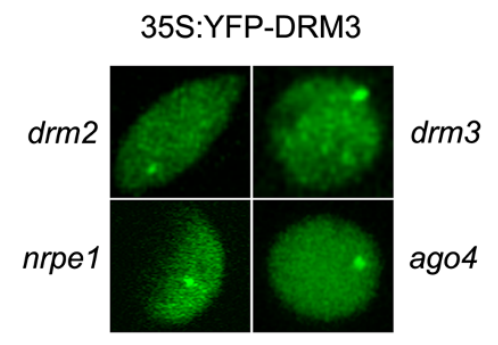

B)

D)
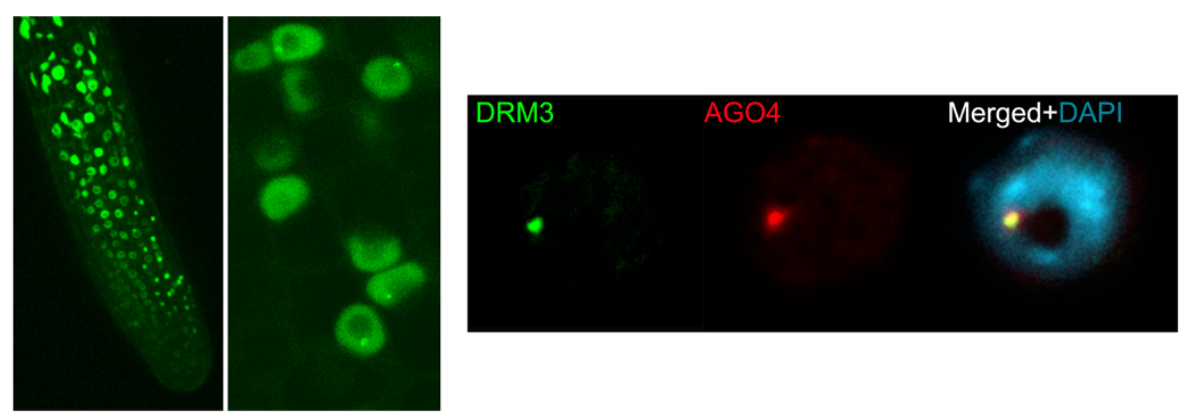

Figure 3 DRM3 colocalizes with AGO4 in a nuclear domain in the nucleolar periphery. (A) Methylation Sensitive PCR showing that the 35S: YFP-DRM3 transgene rescues the methylation phenotype of drm3. (B) Confocal microscope inspection showing transgene expression throughout the root (left panel) and the preferential accumulation of 35S:YFP-DRM3 in a round focus in the nucleolar periphery (right panel). (C) Confocal projections of root meristem nuclei indicate that interphase distribution of DRM3 does not depend on Pol V, AGO4 or DRM2 activity. (D) Immunolocalization of AGO4 (red) and DRM3 (green) show colocalization of both proteins in the smRNA perinucleolar body $(>98 \% ; n=85)$. DNA counterstained with DAPI (blue). $\star-500$ bp. 
DDM1 [31,32]. Within the nucleus, heterochromatin is usually visible as DAPI (4',6-diamidino-2-phenylindole)bright domains - the chromocenters - enriched in repetitive sequences [33,34]. In $d d m 1$ and met 1 nuclei, a reduction in the size of these chromocenters is observed as a result of the dispersion of pericentromeric sequences [33].

We evaluated whether DNA methylation regulated by DRM3 is correlated with spatial organization of heterochromatin within the nucleus. To that end, we used DAPI staining and Fluorescence In Situ Hybridization (FISH) to characterize the interphase organization of heterochromatic repeats in the drm3 mutant background, when compared to met1, cmt3 and drm2 loss of function mutants (Figure 4). First, we measured the chromocenter fraction [35] in met1, cmt3, drm 2 and drm3. With the exception of met1, all analyzed DNA methyltransferase mutant lines displayed similar heterochromatin content as WT (not shown, see also Figure 3), indicating that DRM3-dependent cytosine methylation is not required for heterochromatin assembly during interphase. These observations are in agreement with previously published results showing that heterochromatin compaction is mainly dependent on MET1 activity [33,36] and not on DRM2 [19].

Second, we used FISH with gene-specific probes to characterize the regulatory role of DRM3 in specific heterochromatic regions' interphase organization (Figure 4). A. thaliana has four NOR loci located at the tips of chromosomes 2 and 4 [37,38]. However, during interphase, NORs usually associate [35]; as a result, upon FISH analysis with a $45 \mathrm{~S}$ rRNA DNA probe, three condensed foci, enclosed within chromocenters, were cytologically visible at the nucleolar periphery of the majority of the nuclei

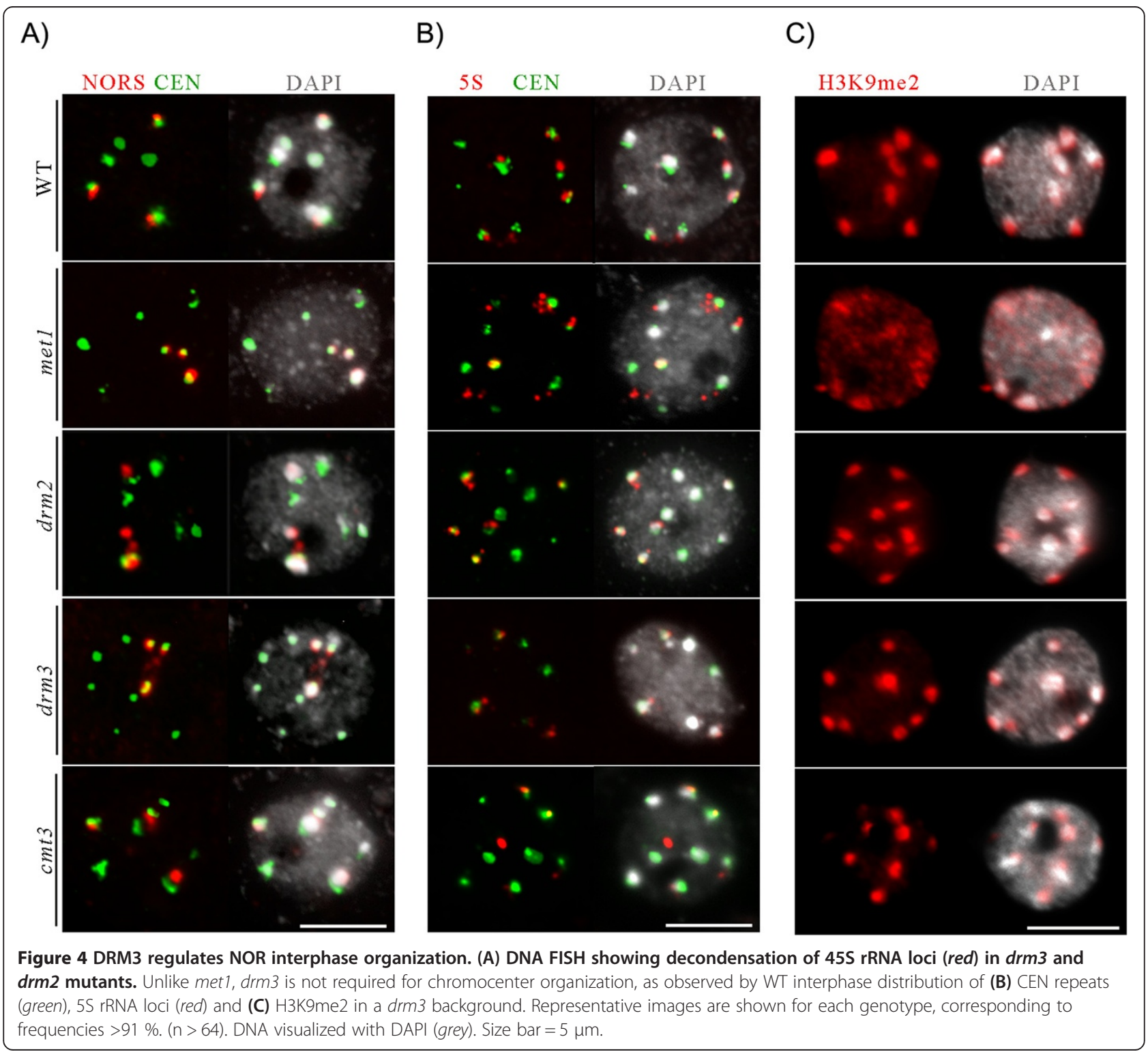


(65\%; Figure 4A). In contrast, in both $d r m 3$ and drm $2 \mathrm{mu}-$ tant backgrounds, more nuclei with four NOR foci were observed ( $43 \%$ and $45 \%$, respectively) relative to WT (30\%) (see also Additional file 1: Table S1). In addition, the NOR's FISH signals were smaller compared to WT nuclei, and partially located inside the nucleolus in both $d r m 2$ and drm3 null mutant backgrounds (red signals, Figure 4A, arrows). Upon FISH analysis with the $45 \mathrm{~S}$ rRNA-specific probe, we observed no loss of association between NOR loci and the chromocenter in met 1 or $c m t 3$ mutants, nor foci decondensation.

In A. thaliana WT, 5S ribosomal DNA (rDNA) loci are located in the pericentromeric heterochromatin of chromosomes 3, 4 and 5 [39]. Both major and some minor 5S rRNA species from chromosomes 4 and 5 are expressed, whereas the genes on chromosome 3 are not [40]. It has been previously demonstrated that in leaf nuclei of mature WT plants, the transcribed fraction of $5 \mathrm{~S}$ rDNA forms loops that emanate from chromocenters in a DDM1- and MET1dependent manner $[41,42]$. Our FISH analysis showed that 5S rRNA loci in cmt3, drm3 and drm2 displayed a WT interphase organization pattern, in contrast to the altered pattern observed in met1 (Figure 4B; Additional file 1: Table S1), as previously reported [42]. These results demonstrate that, like DRM2, DRM3-directed methylation is not required for $5 \mathrm{~S}$ rRNA loci interphase organization [43]. Likewise, FISH with probes specific to other heterochromatic repeat regions, like the 180 -bp centromere repeats, did not detect an altered interphase organization pattern in $d r m 3$ and $d r m 2$ mutant backgrounds (red signals, Figure 4B; Additional file 1: Table S2).

Immunofluorescence analysis using antibodies specific to heterochromatic marks such as H3K9me2 (Figure 4C) and DNA methylation (not shown) revealed that drm3, $d r m 2$ and $c m t 3$ nuclei display a WT nuclear organization, in which the H3K9me2 marks are enclosed in the chromocenters. In contrast, met1 displayed a disrupted nuclear organization, with the H3K9me2 heterochromatic modification no longer confined to the chromocenters (Figure 4C; Additional file 1: Table S3).

In conclusion, DRM3 and DRM2 are both required for NOR loci nuclear organization but not for overall periand centromeric heterochromatin organization, in contrast to MET1. Our cytological observations strengthen the hypothesis that DRM3 acts together with DRM2 in RdDM and does not contribute to MET1 activity in the spatial assembly of pericentromeric heterochromatin.

\section{Materials \& Methods}

Plant material and generation of transgenic lines

Loss of function mutants used in this study included ago4-1 (At2g27040), provided by Steve Jacobsen and in Landsberg background; nrpd1-3 (At1g63020); nrpe1-11 (At2g40030); met1-1 (At5g49160), provided by Eric Richards; cmt3-11 $t$ (SALK_148381) (At1g69770); rdm1-1 (At3g22680) provided by Jian-Kang Zhu; drm3-1, drm3-2 (At3g17310) [10]; and drm2-2 (At5g14620); all in Columbia ecotype (Col-0) background. Plants were grown in long-day conditions in a controlled environment $\left(16 \mathrm{~h}\right.$ light $/ 8 \mathrm{~h}$ dark: $\left.22^{\circ} \mathrm{C}\right)$ on soil or Murashige and Skoog medium with Gamborg vitamins (Sigma).

The full-length coding region of DRM3 was amplified by PCR and cloned into the pEarleyGate104 plant transformation vector using Gateway methodology (Invitrogen), as described previously [44]. The transgenic construct was subsequently transformed into the desired genetic background by the floral dip method [45].

\section{Fluorescence in situ hybridization (FISH),} immunolocalization and microscopy

Leaf nuclei spreads were prepared according to [21]. Probe labeling for FISH was as follows: 45S rRNA probe was labeled with Biotin 16-dUTP following the Nick Translation Mix (Roche) protocol with the pAt.2 [46] plasmid as template; $5 \mathrm{~S}$ rRNA (in pCT4.2) and $180 \mathrm{bp}-\mathrm{CEN}$ (in pARR20.1) probes were labeled by PCR with Biotin 16-dUTP and Digoxigenin-11-dUTP (Roche), respectively. FISH was performed as previously described [47] with a hybridization stringency of $81 \%$ ( $50 \%$ formamide: $2 \times \mathrm{SSC}-$ at $37^{\circ} \mathrm{C}$ ). Biotin-labeled probes were detected with streptavidin-Cy3 conjugate (1:300, Life Technologies). Digoxigenin-labeled probes were detected using mouse anti-digoxigenin antibody (1:250, Roche) followed by incubation with antimouse Alexa488 (Life Technologies). Immunolocalization of AGO4 was performed by making use of an antibody raised in rabbit against the native protein [16]. Immunofluorescence to detect $\mathrm{H} 3 \mathrm{~K} 9 \mathrm{me} 2$ was performed with an anti-dimethyl-Histone H3 (Lys9) antibody (Millipore), as previously described [19]. DNA was counterstained with ProlongGold ${ }^{\diamond}$ antifade reagent with DAPI (Life Technologies). Preparations were inspected using a Zeiss AxioSkop2 mot plus upright microscope equipped with an AxioCam MRm camera. 35S:YFP-DRM3 nuclear localization was evaluated by live cell imaging using a Nikon A1 confocal microscope. Images were processed with AxioVision (Zeiss) and Adobe Photoshop software (Adobe Systems).

\section{DNA methylation analysis}

DNA from 2-week-old leaves was extracted with NucleoSpin ${ }^{\odot}$ Plant II (Macherey-Nagel), according to the manufacturer's instructions. For MSP assays, $500 \mathrm{ng}$ of DNA were digested with the selected restriction enzyme. Reactions without the selected restriction enzyme were also performed to serve as an input control. PCR was subsequently used to evaluate the cytosine methylation status at the targeted positions (Additional file 1: Table S4). Bisulfite conversion was performed with an EZ DNA Methylation- 
Lightning $\mathrm{Kit}^{\mathrm{ta}}$ (Zymo Research), with an input of $600 \mathrm{ng}$ gDNA per sample. Modification efficiency was assessed by PCR amplification with a control primer pair [48]. Amplicons were generated from the target sequences, gel purified, cloned into pGEM ${ }^{-}$-T Easy (Promega) and transformed into bacteria. At least 10 individual clones were sequenced for each genotype per target sequence and sequencing results were analyzed with CyMATE [48]. See Additional file 1: Table S4 for a complete list of primers.

\section{Small RNA blots and transcriptional analysis}

RNA was extracted from leaf tissue of 2-week-old plants following the enrichment procedure for smRNAs of the mirVana $^{\text {ma }}$ miRNA Isolation Kit (Ambion). The high molecular RNA fraction was digested with TURBO ${ }^{\text {th }}$ DNAse (Ambion). One $\mu$ g of RNA was subsequently used for cDNA synthesis with Maxima Reverse Transcriptase (Thermo Scientific) and used as a template in RT-PCR analysis (see Additional file 1: Table S4).

For smRNA analysis, $10 \mu \mathrm{g}$ of the low molecular weight fraction were separated on a $20 \%$ polyacrylamide denaturing gel (7 M urea; $50 \mathrm{mM} \mathrm{TBE}$ ) and transferred on a semidry blotter, $2.0 \mathrm{~mA} / \mathrm{cm}^{2}$ for $2 \mathrm{~h}$, to a MAGNACHARGE nylon membrane $(0.22 \mu \mathrm{m})$ (GE Osmonics). RNA probes were radioactively labeled with a mirVana ${ }^{\text {tm }}$ miRNA Probe Construction Kit (Ambion) according to manufacturer's instructions (Additional file 1: Table S4). RNA hybridization was performed as previously described [49].

\section{Additional file}

Additional file 1: Figure S1. Cymate (cymate.org) output file showing methylation status of cytosines in MEA-ISR loci for the analyzed mutant lines. Red arrows highlight amplicons displaying partial loss of CNN methylation relative to WT. Figure S2. Mapping and comparison of cytosine methylation frequency of AtSNI repeats between WT Col-0, nrpe1, drm2, drm3 and drm2/ drm3 double mutant lines by bisulfite sequencing. Figure S3. (A) AGO4 interphase localization is not dependent of DRM3. No alteration of the immunolocalization patterns of AGO4 in WT and drm3 mutant line was observed. (B) Confocal projection showing DRM3-YFP localizing to the small RNA processing center. DRM3-YFP localization is not disrupted in a drm2 mutant background (compare left panels). Likewise, no alteration to the nucleoplasmic DRM2-GFP interphase localization was observed in a drm3 background relative to WT (compare right panels). Table S1. - Frequency (\%) of nuclei displaying different numbers of NORs in different DNA methylation mutant backgrounds. Table S2. - Frequency (\%) of nuclei displaying different numbers of centromere foci during interphase in DNA methyltranferase mutants. Table S3. - Frequency (\%) of nuclei displaying co-localization of H3K9met immunostaining signals with chromocenters in DNA methyltransferease mutants. Table S4. Primer List. Supplementary references.

\section{Competing interests}

The authors declare that they have no competing interests.

\section{Authors' Contributions}

EH developed the drm3 genetic mutant. JYK developed the DRM3-YFP transgenic line. PCN performed all experiments for Figures 1,2 and 3. OP performed all experiments for Figure 4. PCN and OP designed the experiments and wrote the manuscript. All authors read and approved the final manuscript.

\section{Acknowledgements}

This work was supported by start-up funds from the University of New Mexico.

\section{Author details}

${ }^{1}$ Department of Biology, University of New Mexico, MSC03 2020, 1 University of New Mexico, Albuquerque, NM 87131, USA. '2Department of Biology, Washington University in St. Louis, 1 Brookings Drive, St. Louis, MO 63130, USA. ${ }^{3}$ Present address: Shanghai Center for Plant Stress Biology, Chinese Academy of Sciences, 3888 Chenhua Road, Shanghai 201602, P. R. China.

Received: 16 June 2014 Accepted: 29 September 2014 Published: 14 October 2014

\section{References}

1. Law JA, Ausin I, Johnson LM, Vashisht AA, Zhu JK, Wohlschlegel JA, Jacobsen SE: A protein complex required for polymerase $\mathrm{V}$ transcripts and RNA- directed DNA methylation in Arabidopsis. Curr Biol 2010, 20:951-956

2. Springer NM, Kaeppler SM: Evolutionary divergence of monocot and dicot methyl-CpG-binding domain proteins. Plant Physio/ 2005, 138:92-104.

3. Kankel MW, Ramsey DE, Stokes TL, Flowers SK, Haag JR, Jeddeloh JA, Riddle NC, Verbsky ML, Richards EJ: Arabidopsis MET1 cytosine methyltransferase mutants. Genetics 2003, 163:1109-1122.

4. Lindroth AM, Cao X, Jackson JP, Zilberman D, McCallum CM, Henikoff S, Jacobsen SE: Requirement of CHROMOMETHYLASE3 for maintenance of CpXpG methylation. Science 2001, 292:2077-2080.

5. Stroud H, Do T, Du J, Zhong X, Feng S, Johnson L, Patel DJ, Jacobsen SE: Non-CG methylation patterns shape the epigenetic landscape in Arabidopsis. Nat Struct Mol Biol 2014, 21:64-72.

6. Zemach A, Kim MY, Hsieh PH, Coleman-Derr D, Eshed-Williams L, Thao K, Harmer SL, Zilberman D: The Arabidopsis nucleosome remodeler DDM1 allows DNA methyltransferases to access $\mathrm{H} 1$-containing heterochromatin. Cell 2013, 153:193-205.

7. Jullien PE, Susaki D, Yelagandula R, Higashiyama T, Berger F: DNA methylation dynamics during sexual reproduction in Arabidopsis thaliana. Curr Biol 2012, 22:1825-1830.

8. Cao X, Jacobsen SE: Role of the arabidopsis DRM methyltransferases in de novo DNA methylation and gene silencing. Curr Bio/ 2002, 12:1138-1144.

9. Cao X, Aufsatz W, Zilberman D, Mette MF, Huang MS, Matzke M, Jacobsen SE: Role of the DRM and CMT3 methyltransferases in RNA-directed DNA methylation. Curr Biol 2003, 13:2212-2217.

10. Henderson IR, Deleris A, Wong W, Zhong X, Chin HG, Horwitz GA, Kelly KA, Pradhan S, Jacobsen SE: The de novo cytosine methyltransferase DRM2 requires intact UBA domains and a catalytically mutated paralog DRM3 during RNA-directed DNA methylation in Arabidopsis thaliana. PLOS Genet 2010, 6:e1001182.

11. Naumann U, Daxinger L, Kanno T, Eun C, Long Q, Lorkovic ZJ, Matzke M, Matzke AJ: Genetic evidence that DNA methyltransferase DRM2 has a direct catalytic role in RNA-directed DNA methylation in Arabidopsis thaliana. Genetics 2011, 187:977-979.

12. Law JA, Vashisht AA, Wohlschlegel JA, Jacobsen SE: SHH1, a homeodomain protein required for DNA methylation, as well as RDR2, RDM4, and chromatin remodeling factors, associate with RNA polymerase IV. PLoS Genet 2011, 7:e1002195.

13. Haag JR, Ream TS, Marasco M, Nicora CD, Norbeck AD, Pasa-Tolic L, Pikaard CS: In Vitro Transcription Activities of Pol IV, Pol V, and RDR2 Reveal Coupling of Pol IV and RDR2 for dsRNA Synthesis in Plant RNA Silencing. Mol Cell 2012, 48:811-818.

14. Mi S, Cai T, Hu Y, Chen Y, Hodges E, Ni F, Wu L, Li S, Zhou H, Long C, Chen S, Hannon GJ, Qi Y: Sorting of small RNAs into Arabidopsis argonaute complexes is directed by the 5 ' terminal nucleotide. Cell 2008, 133:116-127.

15. Takeda A, Iwasaki S, Watanabe T, Utsumi M, Watanabe Y: The mechanism selecting the guide strand from small RNA duplexes is different among argonaute proteins. Plant Cell Physiol 2008, 49:493-500.

16. Wierzbicki AT, Ream TS, Haag JR, Pikaard CS: RNA polymerase V transcription guides ARGONAUTE4 to chromatin. Nat Genet 2009, 41:630-634.

17. Zilberman D, Cao X, Jacobsen SE: ARGONAUTE4 control of locus-specific siRNA accumulation and DNA and histone methylation. Proc Natl Acad SCi US A 2003, 299:716. 
18. Jacob Y, Feng S, LeBlanc CA, Bernatavichute $\mathrm{Y}$, Stroud H, Cokus S, Johnson LM, Pellegrini M, Jacobsen SE, Michaels SD: ATXR5 and ATXR6 are H3K27 monomethyltransferases required for chromatin structure and gene silencing. Nat Struct Mol Biol 2009, 16:763-768

19. Pontes $\mathrm{O}$, Costa-Nunes $P$, Vithayathil $P$, Pikaard CS: RNA polymerase $V$ functions in Arabidopsis interphase heterochromatin organization independently of the 24-nt siRNA-directed DNA methylation pathway. Mol Plant 2009, 2:700-710.

20. Huettel B, Kanno T, Daxinger L, Aufsatz W, Matzke AJ, Matzke M: Endogenous targets of RNA-directed DNA methylation and Pol IV in Arabidopsis. EMBO J 2006, 25:2828-2836.

21. Pontes O, Li CF, Nunes PC, Haag J, Ream T, Vitins A, Jacobsen SE, Pikaard CS: The Arabidopsis chromatin-modifying nuclear siRNA pathway involves a nucleolar RNA processing center. Cell 2006, 126:79-92.

22. Ye R, Wang W, Iki T, Liu C, Wu Y, Ishikawa M, Zhou X, Qi Y: Cytoplasmic Assembly and Selective Nuclear Import of Arabidopsis ARGONAUTE4/ siRNA Complexes. Mol Cell 2012, 46:859-870.

23. Li CF, Pontes O, El-Shami M, Henderson IR, Bernatavichute YV, Chan SW, Lagrange T, Pikaard CS, Jacobsen SE: An ARGONAUTE4-containing nuclear processing center colocalized with Cajal bodies in Arabidopsis thaliana. Cell 2006, 126:93-106.

24. Pontes O, Vitins A, Ream TS, Hong E, Pikaard CS, Costa-Nunes P: Intersection of small RNA pathways in Arabidopsis thaliana sub-nuclear domains. PLoS One 2013, 8:e65652.

25. Gao Z, Liu HL, Daxinger L, Pontes O, He X, Qian W, Lin H, Xie M, Lorkovic ZJ, Zhang S, Miki D, Zhan X, Pontier D, Lagrange T, Jin H, Matzke AJ, Matzke M, Pikaard CS, Zhu JK: An RNA polymerase II- and AGO4-associated protein acts in RNA-directed DNA methylation. Nature 2010, 465:106-109.

26. Haag JR, Pontes O, Pikaard CS: Metal A and metal B sites of nuclear RNA polymerases Pol IV and Pol V are required for siRNA-dependent DNA methylation and gene silencing. PLoS One 2009, 4:e4110.

27. Ooi SK, Qiu C, Bernstein E, Li K, Jia D, Yang Z, Erdjument-Bromage H, Tempst $P$, Lin SP, Allis CD, Cheng X, Bestor TH: DNMT3L connects unmethylated lysine 4 of histone $\mathrm{H} 3$ to de novo methylation of DNA Nature 2007, 448:714-717.

28. Jia D, Jurkowska RZ, Zhang $X$, Jeltsch $A$, Cheng $X$ : Structure of Dnmt3a bound to Dnmt3L suggests a model for de novo DNA methylation. Nature 2007, 449:248-251.

29. van Zanten $M$, Tessadori F, Peeters AJ, Fransz P: Shedding light on largescale chromatin reorganization in Arabidopsis thaliana. Mol Plant 2012, 5:583-590.

30. Fransz $\mathrm{P}$, de Jong $\mathrm{H}$ : From nucleosome to chromosome: a dynamic organization of genetic information. Plant J 2011, 66:4-17.

31. Jeddeloh JA, Stokes TL, Richards EJ: Maintenance of genomic methylation requires a SWI2/SNF2-like protein. Nat Genet 1999, 22:94-97.

32. Brzeski J, Jerzmanowski A: Deficient in DNA methylation 1 (DDM1) defines a novel family of chromatin-remodeling factors. J Biol Chem 2003, 278:823-828.

33. Soppe WJ, Jasencakova Z, Houben A, Kakutani T, Meister A, Huang MS, Jacobsen SE, Schubert I, Fransz PF: DNA methylation controls histone H3 lysine 9 methylation and heterochromatin assembly in Arabidopsis. EMBO J 2002, 21:6549-6559.

34. Probst AV, Fransz PF, Paszkowski J, Mittelsten Scheid O: Two means of transcriptional reactivation within heterochromatin. Plant J 2003, 33:743-749.

35. Fransz P, De Jong JH, Lysak M, Castiglione MR, Schubert I: Interphase chromosomes in Arabidopsis are organized as well defined chromocenters from which euchromatin loops emanate. Proc Natl Acad Sci U S A 2002, 99:14584-14589.

36. Fransz $P$, ten Hoopen $R$, Tessadori F: Composition and formation of heterochromatin in Arabidopsis thaliana. Chromosome Res 2006, 14:71-82.

37. Copenhaver GP, Doelling JH, Gens S, Pikaard CS: Use of RFLPs larger than $100 \mathrm{kbp}$ to map the position and internal organization of the nucleolus organizer region on chromosome 2 in Arabidopsis thaliana. Plant J 1995, 7:273-286.

38. Copenhaver GP, Pikaard CS: RFLP and physical mapping with an rDNAspecific endonuclease reveals that nucleolus organizer regions of Arabidopsis thaliana adjoin the telomeres on chromosomes 2 and 4 . Plant J 1996, 9:259-272.

39. Murata M, Heslop-Harrison JS, Motoyoshi F: Physical mapping of the $5 \mathrm{~S}$ ribosomal RNA genes in Arabidopsis thaliana by multi-color fluorescence in situ hybridization with cosmid clones. Plant J 1997, 12:31-37.
40. Cloix C, Tutois S, Yukawa Y, Mathieu O, Cuvillier C, Espagnol MC, Picard G, Tourmente S: Analysis of the 5S RNA pool in Arabidopsis thaliana: RNAs are heterogeneous and only two of the genomic $5 S$ loci produce mature 5S RNA. Genome Re. 2002, 12:132-144.

41. Mathieu O, Jasencakova Z, Vaillant I, Gendrel AV, Colot V, Schubert I, Tourmente S: Changes in 5 S rDNA chromatin organization and transcription during heterochromatin establishment in Arabidopsis. Plant Cell 2003, 15:2929-2939.

42. Vaillant I, Tutois S, Cuvillier C, Schubert I, Tourmente S: Regulation of Arabidopsis thaliana 5S rRNA Genes. Plant Cell Physiol 2007, 48:745-752.

43. Mathieu O, Reinders J, Caikovski M, Smathajitt C, Paszkowski J: Transgenerational stability of the Arabidopsis epigenome is coordinated by CG methylation. Cell 2007, 130:851-862.

44. Earley K, Lawrence RJ, Pontes O, Reuther R, Enciso AJ, Silva M, Neves N, Gross M, Viegas W, Pikaard CS: Erasure of histone acetylation by Arabidopsis HDA6 mediates large-scale gene silencing in nucleolar dominance. Genes Dev 2006, 20:1283-1293.

45. Clough SJ, Bent AF: Floral dip: a simplified method for Agrobacteriummediated transformation of Arabidopsis thaliana. Plant J 1998, 16:735-743.

46. Doelling JH, Gaudino RJ, Pikaard CS: Functional analysis of Arabidopsis thaliana rRNA gene and spacer promoters in vivo and by transient expression. Proc Natl Acad Sci U S A 1993, 90:7528-7532.

47. Pontes O, Lawrence RJ, Neves N, Silva M, Lee JH, Chen ZJ, Viegas W, Pikaard CS: Natural variation in nucleolar dominance reveals the relationship between nucleolus organizer chromatin topology and rRNA gene transcription in Arabidopsis. Proc Natl Acad Sci U S A 2003, 100:11418-11423.

48. Hetzl J, Foerster AM, Raidl G, Mittelsten Scheid O: CyMATE: a new tool for methylation analysis of plant genomic DNA after bisulphite sequencing. Plant J 2007, 51:526-536

49. Onodera Y, Haag JR, Ream T, Nunes PC, Pontes O, Pikaard CS: Plant nuclear RNA polymerase IV mediates siRNA and DNA methylation-dependent heterochromatin formation. Cell 2005, 120:613-622.

doi:10.1186/1756-0500-7-721

Cite this article as: Costa-Nunes et al:: The cytological and molecular role of DOMAINS REARRANGED METHYLTRANSFERASE3 in RNAdependent DNA methylation of Arabidopsis thaliana. BMC Research Notes 2014 7:721.

\section{Submit your next manuscript to BioMed Central and take full advantage of:}

- Convenient online submission

- Thorough peer review

- No space constraints or color figure charges

- Immediate publication on acceptance

- Inclusion in PubMed, CAS, Scopus and Google Scholar

- Research which is freely available for redistribution

Submit your manuscript at www.biomedcentral.com/submit
C) BioMed Central 